\title{
BMJ Open Assessment of surgical outcomes of femtosecond laser-assisted in situ keratomileusis in patients with low compliance to postoperative follow-up: a retrospective observational study in a tertiary hospital in China
}

Qing Zhang, ${ }^{1}$ Jing Wang, ${ }^{1}$ Mohan Li, ${ }^{1}$ Xiaohu Ding, ${ }^{2}$ Liming Tao ${ }^{1}$

To cite: Zhang Q, Wang J, Li M, et al. Assessment of surgical outcomes of femtosecond laser-assisted in situ keratomileusis in patients with low compliance to postoperative follow-up: a retrospective observational study in a tertiary hospital in China. BMJ Open 2018;8:e21702. doi:10.1136/ bmjopen-2018-021702

- Prepublication history for this paper is available online. To view these files, please visit the journal online (http://dx.doi org/10.1136/bmjopen-2018021702).

Received 14 March 2018 Revised 18 September 2018 Accepted 21 September 2018

A) Check for updates

(C) Author(s) (or their employer(s)) 2018. Re-use permitted under CC BY-NC. No commercial re-use. See rights and permissions. Published by BMJ.

${ }^{1}$ Department of Ophthalmology, The Second Affiliated Hospital of Anhui Medical University, Hefei, China

${ }^{2}$ State Key Laboratory of Ophthalmology, Zhongshan Ophthalmic Center, Sun Yat-sen University, Guangzhou, China

Correspondence to

Dr Liming Tao;

taolimingchina@163.com

\section{ABSTRACT}

Objectives Poor follow-up after femtosecond laserassisted in situ keratomileusis (FS-LASIK) is common in general clinical practice. We aimed to assess the surgical outcomes of patients with poor compliance to FS-LASIK follow-up but who returned to the clinic with additional prompting at a 1-year visit. We also compared their surgical outcomes with those of patients who returned unprompted.

Design Retrospective and observational study. Setting An urban tertiary hospital in China.

Participants We reviewed the medical records of myopic patients who underwent binocular FS-LASIK. These patients were all recommended, but not compulsively required, to return for termly postoperative examinations including measurement of uncorrected distance visual acuity (UDVA), refraction and assessment of complications. According to records of follow-up visits, 1009 eligible patients were categorised as follows: (1) 124 who returned unprompted at the 1-year visit (group 1) and (2) 885 lost to follow-up at the 1-year visit. We randomly selected and called back 105 (group 2) out of the 885 patients for an extra postoperative examination.

Results At the 1-year visit, the visual outcomes of the two groups of patients were comparable. No differences in postoperative UDVA were found between the two groups $(-0.02 \pm 0.06$ logarithm of the minimum angle of resolution (logMAR) and $-0.02 \pm 0.05$ logMAR for groups 1 and 2 , respectively, $p=0.175$ ). Patients in group 2 showed greater hyperopic dioptres than patients in group $1(0.37 \pm 0.59 \mathrm{D}$ vs $-0.29 \pm 0.69, p<0.0001)$. No visionthreatening complications were observed in either group of patients.

Conclusions The visual and refractive outcomes of patients who were lost to follow-up after FS-LASIK surgery were good and comparable to those who returned unprompted. The results indicated that rigorous postoperative follow-up may be unnecessary in general clinical practice, except for patients who are at a high risk for postoperative complications.
Strengths and limitations of this study

$\Rightarrow$ Femtosecond laser-assisted in situ keratomileusis (FS-LASIK) is the most common refractive surgery in China.

$\Rightarrow$ However, no study has explored the compulsory follow-up schedule other than limited expert consensus without evidence from clinical research data.

$\Rightarrow$ Our study demonstrated for the first time that rigorous postoperative follow-up may be unnecessary in general clinical practice.

$\Rightarrow$ A limitation of this study is that other aspects of postsurgery visual outcomes, including contrast sensitivity function and high-order aberration, were not assessed.

$\Rightarrow$ The study was limited by its retrospective nature.

$\Rightarrow$ Moreover, the sample size was relatively small.

\section{INTRODUCTION}

Refractive surgery is becoming increasingly popular for myopia correction, particularly in young adults. ${ }^{1}$ Among all surgical types for refractive correction, femtosecond laserassisted in situ keratomileusis (FS-LASIK) is proposed as a good alternative. ${ }^{2-4}$ The refraction and visual outcomes of FS-LASIK are generally satisfactory, ${ }^{5-7}$ and the occurrence of vision-threatening complications is low. ${ }^{6}$

In addition to skilled surgeons and adequate equipment, the surgical success of FS-LASIK also depends on appropriate postoperative monitoring. A consensus has been reached that patients who undergo refractive surgery should be revisited termly until 1 year postoperation $^{8}$ because vision-threatening complications may occur and timely resolution can substantially improve vision. ${ }^{9-11}$ Nevertheless, postoperative follow-up rates can be low in general clinical practice because 
of the inconvenience posed to patients and a failure to communicate the benefits of returning. A comparison of surgical outcomes between returning patients and those who are lost to follow-up is required to reinforce calls for more improved compliance or, alternatively, less restrictive follow-up schedules. However, to the best of our knowledge, studies are lacking on the surgical outcomes of patients who return unprompted and those who return after further exhortation.

In this study, we aimed to assess whether the surgical outcomes of FS-LASIK patients who returned unprompted at a 1-year follow-up examination are comparable to those of patients who returned with additional prompting, and to provide evidence for appropriate schedules for FS-LASIK follow-up in general clinical practice.

\section{METHODS}

\section{Study design and participants}

This study retrospectively reviewed consecutive myopic patients treated with binocular FS-LASIK at the Second Affiliated Hospital of Anhui Medical University, from January 2014 to March 2016. Myopic patients, aged $\geq 18$ years, with spherical myopia $<12 \mathrm{D}$, astigmatism $<6 \mathrm{D}$ and a corrected distant visual acuity (CDVA) $\geq 0.1$ logarithm of the minimum angle of resolution (logMAR) units in both eyes were enrolled in the study. Other inclusion and exclusion criteria, including a stable refractive error, a minimum calculated postoperative residual stromal bed thickness of $250 \mu \mathrm{m}$, and exclusion of systemic and localised ocular disorders, were in concert with the consensus reached in 2015.

A total of 1009 eligible patients were found. For all patients, routine preoperative assessments were performed, including uncorrected distance visual acuity (UDVA) measurement, CDVA measurement, autokeratometry and autorefractometry (Topcon KR-1, Tokyo, Japan), manifest and cycloplegic refraction, axial length measurement (Zeiss, IOLMaster, Meditec, Jena, Germany), corneal topography and central corneal thickness measurement (Pentacam; OculusOptikgeräte, Wetzlar, Germany), slit-lamp biomicroscopy, non-contact intraocular pressure measurement (Topcon, Tokyo, Japan), dilated fundus examination and binocular function evaluation. The percentage of tissue altered (PTA) was calculated using the data of central corneal thickness and the estimated flap thickness and ablation thickness. FS-LASIK was performed binocularly. All patients were recommended to revisit the clinic for postoperative examinations at 1 day, 7 days, 1 month, 3 months, 6 months and 1 year after the surgery. Patients with a high PTA were informed of the high risk of postoperative keratectasia and a rigorous follow-up regimen was suggested. However, termly returning was not mandatory. At every postoperative visit, UDVA measurement, autorefractometry, intraocular pressure measurement and slit-lamp examination were conducted. Any postoperative complications detected at follow-up visits were recorded and resolved.

All eligible patients were stratified into two categories according to their medical records: (1) people who returned unprompted for the 1-year follow-up visit, defined as a return to the clinic within 9-15 months postoperation without additional prompting; and (2) people who were lost to follow-up from the 1-year visit, defined as a failure to return for the 1-year follow-up visit and the subsequent postoperative examinations. Eight patients who returned to the clinic unprompted after 15 months postoperation (median 24 months, 25th and 75th percentile: 20 and 26 months) were categorised into the group of patients who returned unprompted. Thus, this procedure revealed that 124 patients returned unprompted for the 1-year visit and 885 patients were lost to follow-up from the 1-year visit.

We randomly sampled 124 of the 885 patients identified as having lost to follow-up at the 1-year visit. Additional prompting was provided in February 2017 to encourage an extra postoperative visit by using telephone calls and transport subsidies; a total of 105 patients $(84.6 \%)$ returned. The same postoperative evaluations were performed to assess the surgical outcomes of these patients. A questionnaire regarding the reasons for not returning was administered during an in-person interview.

\section{Patient and public involvement}

No patient or member of the public was involved in the study design, or in the conduct of the study.

\section{Statistical analysis}

Data from the right eye were used for statistical analysis. Preoperative characteristics and follow-up rates were compared between patients who returned unprompted and those who were lost follow-up at the 1-year visit using the t-test and $\chi^{2}$ test where appropriate. The follow-up rates of these two groups of patients were calculated at different postoperative time points and compared using the $\chi^{2}$ test. To examine potential selective bias, comparisons were also performed between the patients who returned with additional prompting and the remainder of the patients who were lost to follow-up at the 1-year visit.

To determine the surgical outcomes, postoperative UDVA was divided into four categories (UDVA=-0.1, 0.0 0.1 and $0.2 \log$ MAR) and the proportions of patients with different UDVAs were compared between the patients who returned unprompted (group 1) and those who returned with additional prompting (group 2) using the $\chi^{2}$ test at different follow-up visits. The postoperative autorefraction data of the two groups of patients were compared using t-tests. The extra postoperative examinations of the 105 patients in group 2 were treated as the surgical outcome of the 1-year visit and compared with those of the patients in group 1 because differences in the length of the postoperative period were thought acceptable for comparison for statistical significance (median and 25th and 75th percentile for group 1 versus group 2: 1.01 (0.99 to 1.06 ) vs 1.69 ( 1.47 to 2.31 ) years, $\mathrm{p}<0.001$, $\chi^{2}$ test). 
Table 1 Demographic and preoperative characteristics of patients who returned unprompted and those lost to follow-up at the 1-year postoperative visit

\begin{tabular}{|c|c|c|c|}
\hline & Patients who returned unprompted & Patients lost to follow-up & P values \\
\hline Eyes & 124 & 885 & \\
\hline Age (years) & $24.3 \pm 4.5$ & $23.0 \pm 4.2$ & 0.0013 \\
\hline UDVA (logMAR) & $1.21 \pm 0.38$ & $1.18 \pm 0.41$ & 0.3794 \\
\hline CDVA (logMAR) & $-0.01 \pm 0.04$ & $-0.01 \pm 0.04$ & 0.7704 \\
\hline Cylinder (D) & $-0.71 \pm 0.54$ & $-0.76 \pm 0.63$ & 0.3935 \\
\hline SE (D) & $-5.60 \pm 1.89$ & $-5.06 \pm 1.88$ & 0.0032 \\
\hline Axial length (mm) & $25.7 \pm 1.0$ & $25.6 \pm 1.0$ & 0.5673 \\
\hline СCT $(\mu \mathrm{m})$ & $534.4 \pm 27.3$ & $537.1 \pm 32.1$ & 0.3710 \\
\hline \multicolumn{4}{|l|}{ PTA } \\
\hline \multicolumn{4}{|c|}{$\begin{array}{l}\text { Data were compared using t-tests and are expressed as the mean } \pm \text { SD deviation except when otherwise stated. } \\
{ }^{*} \text { Compared using the } \chi^{2} \text { test. } \\
\text { CCT, central corneal thickness; CDVA, corrected distance visual acuity; logMAR, logarithm of the minimum angle of resolution; PTA, } \\
\text { percentage of tissue altered; SE, spherical equivalent; UDVA, uncorrected distance visual acuity. }\end{array}$} \\
\hline
\end{tabular}

All statistical analyses were performed with STATA V.12.0, and $\mathrm{p}<0.05$ was considered statistically significant.

\section{RESULTS}

A total of 1009 patients were found eligible. The median age was 22 years (range $18-35$ years) and $556(55.1 \%)$ were males. All of the preoperative examinations, including the CDVA measurement and binocular function evaluation, were normal in all of the participants. Of the 1009 eligible patients, 124 (12.3\%) returned unprompted for the 1-year postoperative examination. The demographic and preoperative characteristics of the patients who returned unprompted and those lost to follow-up at the 1-year visit are shown in table 1. In general, people who were lost to follow-up at the 1-year visit tended to be younger, male and with lower myopic spherical dioptres. The mean PTA was slightly greater among the patients who returned unprompted $(36.3 \% \pm 4.6 \%)$ than those lost to follow-up at the 1-year visit $(35.4 \% \pm 4.9 \%)$. However, the difference was small and not statistically significant $(\mathrm{p}=0.0896)$. The percentage of patients with a PTA $>40 \%$ did not differ between the patients who returned unprompted and those lost to follow-up at the 1-year visit. Differences in UDVA, CDVA, astigmatism, axial length and central corneal thickness between the two groups of patients were not statistically significant.

Follow-up rates at different time points are shown in table 2. Compared with the patients who returned unprompted for the 1-year visit, those lost to follow-up at the 1-year visit showed lower returning rates at every postoperative visit except for the 1-day examination.
Among the 885 patients lost to follow-up at the 1-year visit, 105 were called back for an extra postoperative evaluation. Compared with the remaining patients, these 105 patients were more likely to be female $(\mathrm{p}=0.011)$, whereas their age, preoperative characteristics, and visual and refractive outcomes at all postoperative visits showed no difference (table 3). Among these 105 patients, the most frequent reason for not returning was participating in the army $(41,39.0 \%)$, followed by being satisfied with the postoperative vision $(33,31.4 \%)$. Other common reasons included being far from the hospital $(15,14.3 \%)$ and being pregnant $(8,7.6 \%)$.

Compared with the patients in group 1, those in group 2 had better UDVAs at the 3-month and 6-month visits (3-month visit: $-0.04 \pm 0.06$ vs $-0.02 \pm 0.05, \mathrm{p}=0.03$; 6 -month visit: $-0.06 \pm 0.05$ vs $-0.03 \pm 0.05, \mathrm{p}<0.01)$. At the

Table 2 Postoperative follow-up rates of patients who returned unprompted at the 1-year visit and those lost to follow-up at the 1-year visit

\begin{tabular}{lllc}
\hline & $\begin{array}{l}\text { Patients who } \\
\text { returned } \\
\text { unprompted } \\
(\mathbf{n = 1 2 4 )} \mathbf{( \% )}\end{array}$ & $\begin{array}{l}\text { Patients lost } \\
\text { to follow-up } \\
(\mathbf{n}=885)(\%)\end{array}$ & P values \\
\hline 1 day & 100 & 99.4 & 0.401 \\
\hline 7 days & 100 & 90.1 & $<0.001$ \\
\hline 1 month & 100 & 69.5 & $<0.001$ \\
\hline 3 months & 100 & 39.6 & $<0.001$ \\
\hline 6 months & 83.1 & 19.6 & $<0.001$ \\
\hline 1 year & 100 & 0 & $<0.001$ \\
\hline
\end{tabular}


Table 3 Demographic, preoperative and postoperative characteristics of patients who returned with additional promoting and the remainder who were lost to follow-up at the 1-year visit

\begin{tabular}{|c|c|c|c|}
\hline & $\begin{array}{l}\text { Patients who returned with } \\
\text { additional prompting }\end{array}$ & $\begin{array}{l}\text { Remainder of patients lost to follow- } \\
\text { up at the } 1 \text {-year visit }\end{array}$ & $P$ values \\
\hline \multicolumn{4}{|c|}{ Preoperative characteristics } \\
\hline Eyes & 105 & 780 & - \\
\hline Age (years) & $23.5 \pm 4.3$ & $22.9 \pm 4.1$ & 0.178 \\
\hline Male* $(\%)$ & $45.7 \%$ & $58.8 \%$ & 0.011 \\
\hline UDVA (logMAR) & $1.21 \pm 0.39$ & $1.17 \pm 0.42$ & 0.396 \\
\hline CDVA (logMAR) & $-0.01 \pm 0.04$ & $-0.01 \pm 0.04$ & 0.955 \\
\hline Sphere (D) & $-4.72 \pm 1.71$ & $-4.68 \pm 1.85$ & 0.840 \\
\hline Cylinder (D) & $-0.69 \pm 0.60$ & $-0.76 \pm 0.63$ & 0.245 \\
\hline SE (D) & $-5.06 \pm 1.77$ & $-5.06 \pm 1.90$ & 0.997 \\
\hline Axial length $(\mathrm{mm})$ & $25.7 \pm 1.1$ & $25.6 \pm 1.0$ & 0.615 \\
\hline ССT $(\mu \mathrm{m})$ & $539.3 \pm 26.9$ & $536.9 \pm 32.8$ & 0.461 \\
\hline \multicolumn{4}{|l|}{ PTA } \\
\hline Mean (\%) & $35.6 \pm 4.5$ & 35. $4 \pm 4.9$ & 0.7069 \\
\hline$>40 \%{ }^{*}, \%$ & 19.2 & 19.6 & 0.923 \\
\hline \multicolumn{4}{|l|}{1 day postoperation } \\
\hline UDVA (logMAR) & $0.01 \pm 0.06$ & $0.01 \pm 0.06$ & 0.947 \\
\hline Sphere (D) & $0.43 \pm 0.54$ & $0.50 \pm 0.60$ & 0.273 \\
\hline Cylinder (D) & $-0.35 \pm 0.31$ & $-0.36 \pm 0.32$ & 0.376 \\
\hline SE (D) & $0.26 \pm 0.53$ & $0.31 \pm 0.61$ & 0.465 \\
\hline \multicolumn{4}{|l|}{7 days postoperation } \\
\hline UDVA (logMAR) & $-0.01 \pm 0.05$ & $-0.02 \pm 0.05$ & 0.164 \\
\hline Sphere (D) & $0.33 \pm 0.70$ & $0.35 \pm 0.63$ & 0.830 \\
\hline Cylinder (D) & $-0.32 \pm 0.27$ & $-0.32 \pm 0.31$ & 0.952 \\
\hline SE (D) & $0.18 \pm 0.72$ & $0.19 \pm 0.64$ & 0.913 \\
\hline \multicolumn{4}{|c|}{1 month postoperation } \\
\hline UDVA (logMAR) & $-0.01 \pm 0.05$ & $-0.02 \pm 0.02$ & 0.127 \\
\hline Sphere (D) & $0.31 \pm 0.51$ & $0.26 \pm 0.58$ & 0.433 \\
\hline Cylinder (D) & $-0.24 \pm 0.35$ & $-0.21 \pm 0.36$ & 0.452 \\
\hline SE (D) & $0.18 \pm 0.55$ & $0.15 \pm 0.60$ & 0.613 \\
\hline \multicolumn{4}{|c|}{3 months postoperation } \\
\hline UDVA (logMAR) & $-0.01 \pm 0.05$ & $-0.02 \pm 0.05$ & 0.251 \\
\hline Sphere (D) & $0.17 \pm 0.53$ & $0.18 \pm 0.58$ & 0.887 \\
\hline Cylinder (D) & $-0.33 \pm 0.33$ & $-0.29 \pm 0.34$ & 0.426 \\
\hline SE (D) & $0.01 \pm 0.55$ & $0.03 \pm 0.57$ & 0.725 \\
\hline \multicolumn{4}{|c|}{6 months postoperation } \\
\hline UDVA (logMAR) & $-0.01 \pm 0.05$ & $-0.02 \pm 0.05$ & 0.581 \\
\hline Sphere (D) & $0.12 \pm 0.56$ & $0.07 \pm 0.63$ & 0.309 \\
\hline Cylinder (D) & $-0.32 \pm 0.41$ & $-0.31 \pm 0.34$ & 0.912 \\
\hline SE (D) & $-0.04 \pm 0.54$ & $-0.15 \pm 0.62$ & 0.312 \\
\hline
\end{tabular}

${ }^{*}$ Compared using the $\chi^{2}$ test.

CCT, central corneal thickness; CDVA, corrected distance visual acuity; logMAR, logarithm of the minimum angle of resolution; SE, spherical equivalent; PTA, percentage of tissue altered; UDVA, uncorrected distance visual acuity.

1-year visit, the visual outcomes of the two groups were comparable. The mean postoperative UDVA in group 2 was $-0.02 \pm 0.05 \log$ MAR, whereas that in group 1 was
$-0.02 \pm 0.06 \log$ MAR $(p=0.175)$. Figure 1 presents the proportions of patients with different UDVA outcomes in the two groups. Compared with group 1, a greater 


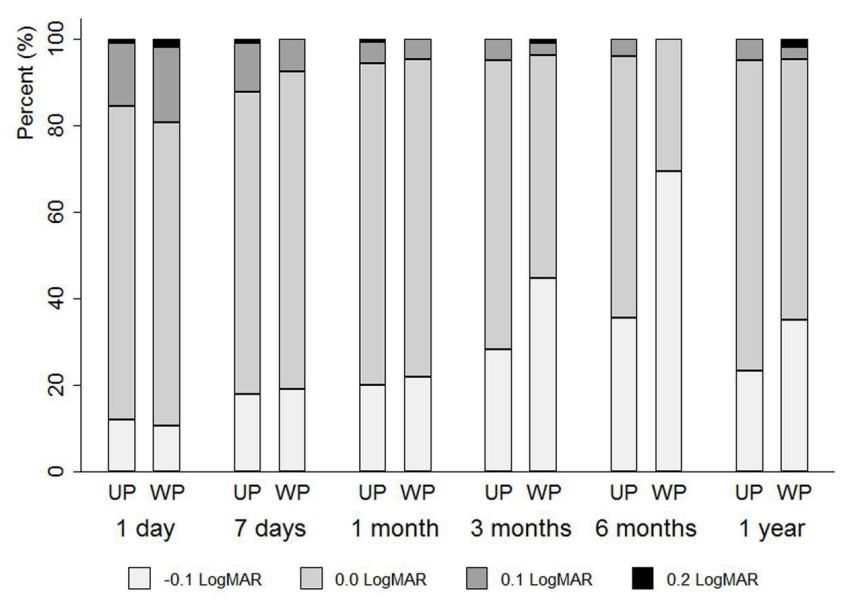

Figure 1 Profiles of the postoperative uncorrected distance visual acuity of the patients who returned unprompted and those who returned with additional prompting. logMAR, logarithm of the minimum angle of resolution; UP, patients who returned unprompted; WP, patients who returned with additional prompting.

proportion of patients in group 2 had UDVAs equal to -0.1 logMAR at the 3-month and 6-month visits (3-month visit: $\chi^{2}=8.3, \mathrm{p}=0.04 ; 6$-month visit: $\left.\chi^{2}=28.0, \mathrm{p}<0.001\right)$. At the 1-year follow-up visit, the difference in the visual outcomes between the two groups had borderline significance $\left(\chi^{2}=6.8, \mathrm{p}=0.07\right)$, with group 2 having a higher proportion of UDVAs equal to $-0.1 \log$ MAR.

Refractive outcomes at the different follow-up visits are shown in figure 2. The spherical equivalent did not differ between groups 1 and 2 at the 1-day, 7-day, 1-month, 3 -month and 6-month visits. At the 1 -year visit, refraction in group 2 exhibited more hyperopic change compared with group 1 (spherical equivalent of patients in group 2 vs group 1: $0.37 \pm 0.59 \mathrm{D}$ vs $-0.29 \pm 0.69, \mathrm{p}<0.0001)$. Of all

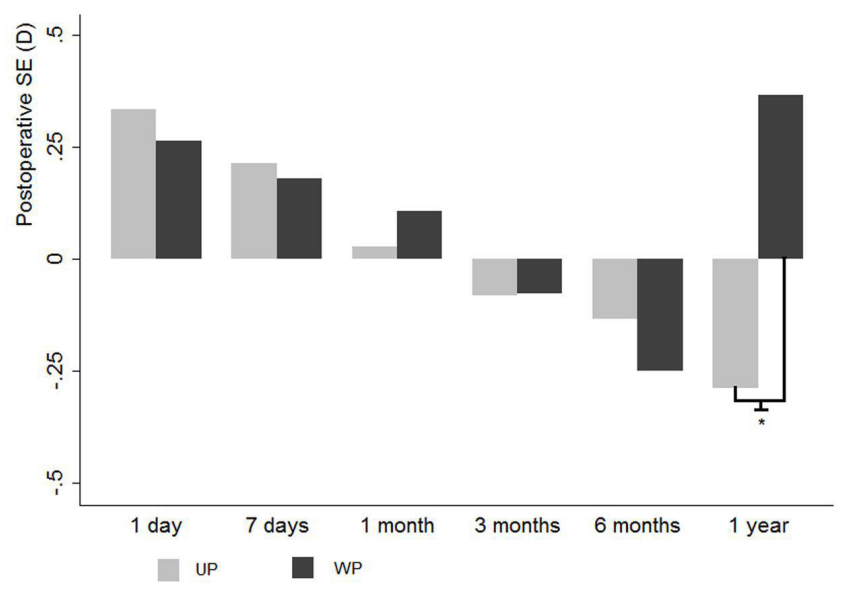

Figure 2 Postoperative refraction of the patients who returned unprompted and those who returned with additional prompting. SE, spherical equivalence; UP, patients who returned unprompted; WP,: patients who returned with additional prompting. ${ }^{*} \mathrm{p}<0.0001$. of the participants in both groups, no vision-threatening complications were detected throughout the follow-up period.

\section{DISCUSSION}

The current study revealed that the visual outcomes of patients who underwent FS-LASIK and returned with additional prompting for the 1-year visit were comparable to those who returned unprompted. Furthermore, refraction tended to be more hyperopic in this group of patients compared with the unprompted patients. The results indicated that the surgical outcomes of patients who were lost to follow-up may be no worse than the patients who returned. In consideration of the high efficacy and safety of FS-LASIK, ${ }^{71} 13$ rigorous postoperative follow-up may be unnecessary in general clinical practice.

FS-LASIK is a satisfactory surgical alternative for refractive correction of myopia. In previous reports, approximately $84 \%-88 \%$ of patients achieved a UDVA of 0.0 logMAR or better after surgery. ${ }^{14} 15$ This figure was approximately $95 \%$ in our study at 1-year postoperation. Good surgical outcomes of FS-LASIK are universally acknowledged to depend on skilled surgeons, adequate equipment and appropriate postoperative monitoring and resolution of surgical complications. At present, evidence-based schedules are lacking for FS-LASIK follow-up visits. Research on refractive surgery typically involves scheduling participants for follow-up visits at 1 day, 7 days, 1 month, 3 months, 6 months and 1 year postoperation. ${ }^{16}$ In China, expert consensus has been achieved with the same recommended follow-up schedules. ${ }^{8}$ However, as recognised in general clinical practice and as reported in the current study, compliance to postoperative follow-up can be very low even when termly follow-up visits are advised.

Determining whether a greater emphasis on compliance to postoperative follow-up is needed is crucial. Our study results indicated that people with younger age, male gender and lower preoperative myopic refractive change tend to be less compliant to postoperative follow-up. Furthermore, a greater proportion of this group of patients had a UDVA of -0.1 logMAR at 3-month and 6-month visits, and their refraction was more hyperopic 1 year after the surgery. Patients lost to follow-up seem to have milder disease severity preoperation and superior visual outcomes postoperation. These characteristics might indicate a lower risk of developing visionthreatening complications after FS-LASIK. Thus, a low postoperative return rate or a less restricted follow-up schedule for FS-LASIK management might be acceptable, considering the high efficacy and safety of the surgery achieved in most clinical settings. Nevertheless, patients who are assessed as having a high risk of postoperative complications should visit the clinic more frequently and individualised follow-up should be scheduled.

In conclusion, our study evaluated the surgical outcomes of FS-LASIK among patients with poor compliance to 
follow-up and found that their surgical outcomes were comparable to those who returned unprompted. The study findings provide evidence for optimising schedules for follow-up visits after FS-LASIK in general clinical practice.

Correction notice The article has been corrected since it was published online. The text "This study retrospectively reviewed consecutive myopic patients treated with binocular FS-LASIK at the Second Affiliated Hospital of Anhui Medical University, from January 2014 to August 2015" has been updated to "This study retrospectively reviewed consecutive myopic patients treated with binocular FSLASIK at the Second Affiliated Hospital of Anhui Medical University, from January 2014 to March 2016.

Contributors Q-Z: data acquisition and analysis, drafting and revising of the manuscript. JW: data acquisition. ML: data acquisition. XH: data analysis and interpretation, manuscript revision. LT: conception and design of the work and manuscript revision. All authors made the decision to submit this manuscript for publication and vouch for the accuracy and completeness of the data and analyses.

Funding The authors have not declared a specific grant for this research from any funding agency in the public, commercial or not-for-profit sectors.

Competing interests None declared.

Patient consent Obtained.

Ethics approval The study was approved by the Ethics Committee of the Second Affiliated Hospital of Anhui Medical University and conducted in accordance with the Declaration of Helsinki.

Provenance and peer review Not commissioned; externally peer reviewed.

Data sharing statement The data sets during the current study are available from the corresponding author on reasonable request.

Open access This is an open access article distributed in accordance with the Creative Commons Attribution Non Commercial (CC BY-NC 4.0) license, which permits others to distribute, remix, adapt, build upon this work non-commercially, and license their derivative works on different terms, provided the original work is properly cited, appropriate credit is given, any changes made indicated, and the use is non-commercial. See: http://creativecommons.org/licenses/by-nc/4.0/.

\section{REFERENCES}

1. Chan C, Lawless M, Sutton G, et al. Small incision lenticule extraction (SMILE) in 2015. Clin Exp Optom 2016;99:204-12.
2. Wang D, Liu M, Chen Y, et al. Differences in the corneal biomechanical changes after SMILE and LASIK. J Refract Surg 2014;30:702-7.

3. Li M, Niu L, Qin B, et al. Confocal comparison of corneal reinnervation after small incision lenticule extraction (SMILE) and femtosecond laser in situ keratomileusis (FS-LASIK). PLoS One 2013;8:e81435

4. Wu D, Wang Y, Zhang L, et al. Corneal biomechanical effects: small-incision lenticule extraction versus femtosecond laserassisted laser in situ keratomileusis. J Cataract Refract Surg 2014;40:954-62.

5. Zhang Y, Shen Q, Jia Y, et al. Clinical Outcomes of SMILE and FS-LASIK used to treat myopia: a meta-analysis. J Refract Surg 2016;32:256-65.

6. Tanna M, Schallhorn SC, Hettinger KA. Femtosecond laser versus mechanical microkeratome: a retrospective comparison of visual outcomes at 3 months. J Refract Surg 2009;25:S668-71.

7. Kezirian GM, Stonecipher KG. Comparison of the IntraLase femtosecond laser and mechanical keratomes for laser in situ keratomileusis. J Cataract Refract Surg 2004;30:804-11.

8. Corneal Group of Ophthalmology Society, Chinese Medical Association. [Consensus of excimer laser cornea refractive surgery]. Zhonghua Yan Ke Za Zhi 2015;51:249-54.

9. Randleman JB, Shah RD. LASIK interface complications: etiology, management, and outcomes. J Refract Surg 2012;28:575-88.

10. Wang B, Zhang Z, Naidu RK, et al. Comparison of the change in posterior corneal elevation and corneal biomechanical parameters after small incision lenticule extraction and femtosecond laserassisted LASIK for high myopia correction. Cont Lens Anterior Eye 2016;39:191-6.

11. Bucci MG, McCormick GJ. Idiopathic peripheral necrotizing keratitis after femtosecond laser in situ keratomileusis. J Cataract Refract Surg 2012;38:544-7.

12. Vestergaard AH, Grauslund J, Ivarsen AR, et al. Central corneal sublayer pachymetry and biomechanical properties after refractive femtosecond lenticule extraction. J Refract Surg 2014;30:102-8.

13. Kymionis GD, Kankariya VP, Plaka AD, et al. Femtosecond laser technology in corneal refractive surgery: a review. J Refract Surg 2012;28:912-20.

14. Lin F, Xu Y, Yang Y. Comparison of the visual results after SMILE and femtosecond laser-assisted LASIK for myopia. J Refract Surg 2014;30:248-54.

15. Ganesh S, Gupta R. Comparison of visual and refractive outcomes following femtosecond laser- assisted lasik with smile in patients with myopia or myopic astigmatism. J Refract Surg 2014;30:590-6.

16. Kim JR, Kim BK, Mun SJ, et al. One-year outcomes of small-incision lenticule extraction (SMILE): mild to moderate myopia vs. high myopia. BMC Ophthalmol 2015;15:59. 